\title{
Advantages of Increasing Writing Temperature in Heat-Assisted Magnetic Recording
}

\author{
T. Kobayashi, Y. Isowaki, and Y. Fujiwara \\ Graduate School of Engineering, Mie Univ., 1577 Kurimamachiya-cho, Tsu 514-8507, Japan
}

\begin{abstract}
The advantages of increasing the writing temperature $T_{\mathrm{w}}$ in heat-assisted magnetic recording (HAMR) are summarized. As $T_{\mathrm{w}}$ increases, the anisotropy constant ratio, which is a new parameter we introduced, can be decreased since the anisotropy constant at the working temperature and the heat-transfer thermal gradient are increased. A relatively thin recording layer (RL) is allowable since the heat-transfer thermal gradient is reduced by increasing the RL thickness because of the adiabatic effect of the RL. Relatively large standard deviations in grain size and anisotropy are permissible since the probability of magnetization reversal is low even for small grains and grains with small anisotropy during the writing period, respectively. A relatively large standard deviation in the Curie temperature is allowable since the heat-transfer thermal gradient is increased. For Fe-Ni-Pt films, the temperature dependence of the anisotropy field is suitable for HAMR when $T_{\mathrm{w}}$ is high. All of the above are advantageous when preparing HAMR media.
\end{abstract}

Key words: heat-assisted magnetic recording, writing temperature, anisotropy constant ratio, standard deviation, temperature dependence

\section{Introduction}

Heat-assisted magnetic recording (HAMR) is a candidate for solving the trilemma problem ${ }^{1)}$ of magnetic recording (MR). HAMR is a recording method in which the medium is heated to reduce the coercivity during the writing period.

We have reported a design method that uses a model calculation for HAMR media ${ }^{2}$. In that paper, we introduced a new parameter, the anisotropy constant ratio $K_{\mathrm{u}} / K_{\text {bulk }}$, which is the intrinsic ratio of film anisotropy constant $K_{\mathrm{u}}$ to bulk $K_{\mathrm{u}}$. Finding a way to increase $K_{\mathrm{u}} / K_{\text {bulk }}$ is a challenging task. Therefore, it is necessary to design a medium with a smaller $K_{\mathrm{u}} / K_{\text {bulk }}$. The dependences of $K_{\mathrm{u}} / K_{\text {bulk }}$ on the grain number per bit $n$, the standard deviation of the grain size $\sigma_{\mathrm{D}}$, the recording layer thickness $h$, and the writing temperature $T_{\mathrm{w}}$ were clarified.

We have subsequently improved our design method, and many relationships between the media design parameters and $K_{\mathrm{u}} / K_{\text {bulk }}$ have been revealed ${ }^{3)}$. The design parameters we examined were the MR method (HAMR and HAMR combined with shingled MR (SHAMR)), $h$, the thermal conductivity of the interlayer 1 (shown in Fig. 2), the light-spot diameter, the heat-spot diameter, the linear velocity, $T_{\mathrm{w}}, n$, and $\sigma_{\mathrm{D}}$. As a result, we found that increasing $T_{\mathrm{w}}$ is only effective for reducing $K_{\mathrm{u}} / K_{\text {bulk }}$ when we compare it with the $K_{\mathrm{u}} / K_{\text {bulk }}$ value calculated using standard parameter values.

The above-mentioned examinations were carried out by changing one parameter. Next, we provided examination results for a combination of more than two parameters $^{4}$. In conclusion, the combinations that can reduce $K_{\mathrm{u}} / K_{\text {bulk }}$ always have SHAMR as one parameter. However, the use of SHAMR degrades the $\mathrm{read} / \mathrm{write}$ performance of the hard drive. Although a lower $T_{\mathrm{w}}$ is better in terms of the heat resistance of the writing head and/or the surface lubricant, increasing $T_{\mathrm{w}}$ appears to be the only way of reducing $K_{\mathrm{u}} / K_{\text {bulk }}$ for HAMR media.

Increasing $T_{\mathrm{w}}$ has many advantages in addition to reducing $K_{\mathrm{u}} / K_{\text {bulk }}$. In this study, we summarize the advantages of increasing $T_{\mathrm{w}}$.

\section{Calculation Conditions}

The medium was assumed to be granular. The arrangement of the grains was not considered. Figure 1 (a) is a schematic illustration of the area near the writing position for HAMR. The writing field $H_{\mathrm{w}}$ is applied to a wide area including the writing position. The circle denoted by $T_{\mathrm{w}}$ is an isotherm of $T_{\mathrm{w}}$, and $d_{\mathrm{w}}$ is the heat-spot diameter. The white regions indicate upward or downward magnetization, and the gray regions indicate the magnetization transition that contains upward and downward magnetization grains. The transition region spreads to adjacent tracks as a result of rewriting operations on the $i$ th track.

$T_{\text {rec }}$ is the maximum temperature ${ }^{3)}$ at which the information on the trailing side can be held during writing. $\Delta x$ is the distance ${ }^{3)}$ from the position of $T_{\mathrm{w}}$ to that of $T_{\text {rec }}$. On the other hand, $T_{\text {adj }}$ is the maximum temperature ${ }^{3)}$ at which the information in adjacent tracks can be held during rewriting. $\Delta y$ is the distance ${ }^{3)}$ from the position of $T_{\mathrm{w}}$ to that of $T_{\text {adj }}$.

The bit area $S$ is fixed, and $S$ is the product of the bit pitch $d_{\mathrm{B}}$ and the track pitch $d_{\mathrm{T}}$. The method for determining $d_{\mathrm{T}} / d_{\mathrm{B}}$ was reported in a previous paper $^{2)}$.

Figure 1 (b) shows the writing-head configuration. It was assumed that the main-pole size of the head is $600 \mathrm{~nm}$ (down-track direction) $\times 300 \mathrm{~nm}$ (cross-track 
direction), and the writing position is located on the trailing side of the main pole. $H_{\text {adj }}$ is the maximum head field ${ }^{3}$ ) that can hold the information under the main pole during rewriting. The maximum temperature under the main pole is $T_{\mathrm{a}}$, which is the maximum working temperature of the hard drive, and was assumed to be $330 \mathrm{~K}$.

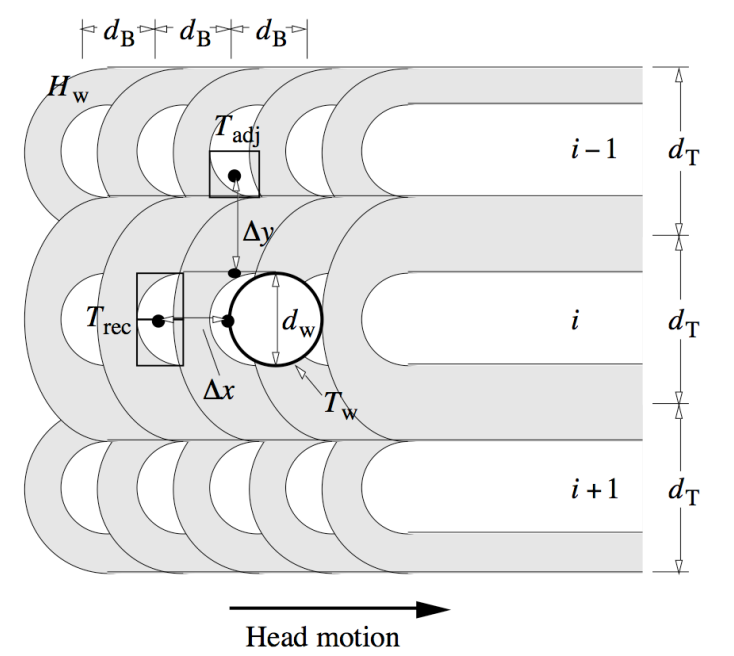

(a)

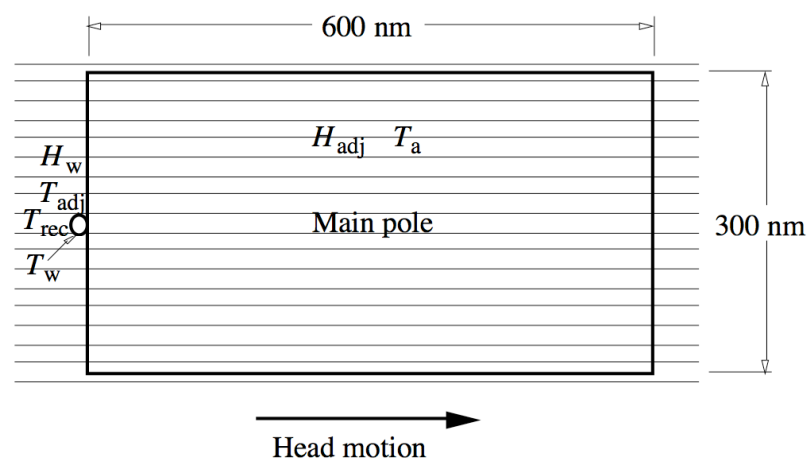

(b)

Fig. 1 Schematic illustrations of (a) writing position and (b) writing-head configuration.

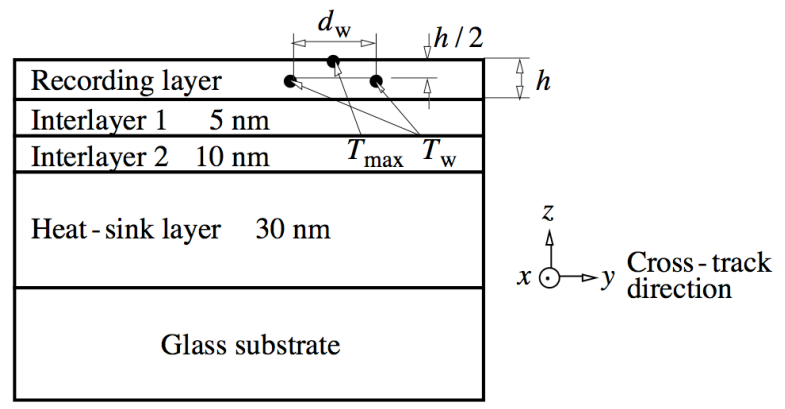

Fig. 2 Medium structure and definition of temperatures.

The standard medium structure is shown in Fig. 2. The standard medium consists of four layers, that is, a recording layer $\mathrm{RL}$ (Fe-Pt base, thickness $h=8 \mathrm{~nm}$ ), an interlayer $1 \mathrm{IL} 1$ ( $\mathrm{MgO}$ base, $5 \mathrm{~nm}$ ), an interlayer 2
IL2 ( $\mathrm{Cr}$ base, $10 \mathrm{~nm})$, and a heat-sink layer HSL $(\mathrm{Cu}$ base, $30 \mathrm{~nm}$ ). The $x, y$, and $z$ axes are the down-track, cross-track, and thickness directions, respectively. $T_{\mathrm{w}}$ is defined at the heat-spot edge and is at the center of the RL layer in the thickness direction. The two positions of $T_{\mathrm{w}}$ in Fig. 2 are at a distance of $d_{\mathrm{w}}$ in the cross-track direction as shown in Fig. 1 (a). $T_{\mathrm{w}}$ and $d_{\mathrm{w}}$ are the design parameters. $T_{\mathrm{w}}$ can be changed by the light power used for heating. $T_{\max }$ is defined as the maximum surface temperature.

Figure 3 (a) shows the heat-transfer thermal gradient $\partial T / \partial x$ for the down-track direction and $\partial T / \partial y$ for the cross-track direction at $T_{\mathrm{w}}$ and the center of the RL layer in the thickness direction as a function of the RL thickness $h . \partial T / \partial x$ and $\partial T / \partial y$ were calculated by a heat-transfer simulation ${ }^{2)}$, and $\partial T / \partial x \approx \partial T / \partial y$ can be seen. The dependence of $T_{\max }$ on $h$ is shown in Fig. 3 (b).

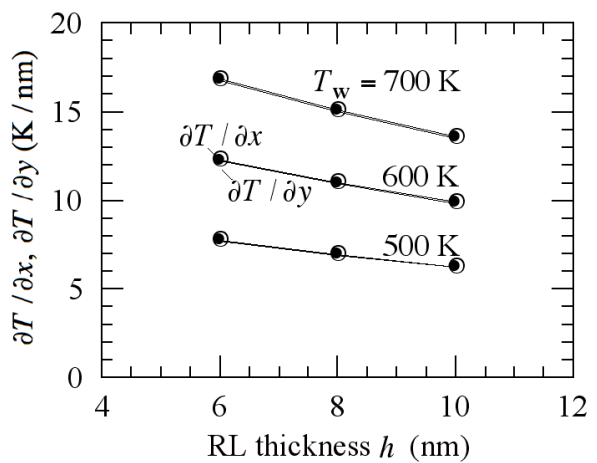

(a)

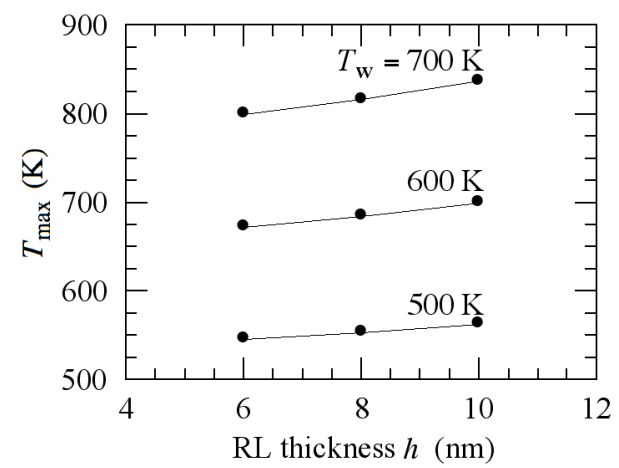

(b)

Fig. 3 (a) Heat-transfer thermal gradients $\partial T / \partial x$ for down-track direction and $\partial T / \partial y$ for cross-track direction, and (b) maximum surface temperature $T_{\max }$ as a function of RL thickness $h$.

The standard parameter values used for the media design are summarized in Table 1 . The standard deviations of the anisotropy constant $\sigma_{\mathrm{K}}$ and the Curie temperature $\sigma_{T \mathrm{c}}$ are newly introduced design parameters.

The HAMR media are designed to obtain the minimum $K_{\mathrm{u}} / K_{\text {bulk }}$ value using the procedure shown in Fig. 4. First, the design parameters and $K_{\mathrm{u}} / K_{\text {bulk }}=$ 1 are set. Four HAMR conditions (1), (2), (3), and (4) are 
examined after determining the composition of the recording layer. If there are margins for all four conditions, $K_{\mathrm{u}} / K_{\text {bulk }}$ can be reduced. When one of the four conditions reaches the limit, the minimum $K_{\mathrm{u}} / K_{\text {bulk }}$ value can be determined. That condition becomes a limiting factor.

Table 1 Standard parameter values used for media design.

\begin{tabular}{l|c}
\hline User areal density (Tbpsi) & 4 \\
Bit area $S\left(\mathrm{~nm}^{2}\right)$ & 140 \\
Effective track width $d_{\mathrm{ET}}(\mathrm{nm})$ & 10 \\
Ambient temperature $T_{\mathrm{a}}(\mathrm{K})$ & 330 \\
\hline Writing temperature $T_{\mathrm{w}}(\mathrm{K})$ & 500 \\
Grain number per bit $n$ (grain / bit) & 4 \\
RL thickness $h(\mathrm{~nm})$ & 8 \\
Standard deviation of grain size $\sigma_{\mathrm{D}} / D_{\mathrm{m}}(\%)$ & 10 \\
Standard deviation of anisotropy $\sigma_{\mathrm{K}} / K_{\mathrm{um}}(\%)$ & 0 \\
Inter - grain exchange coupling $J\left(\mathrm{erg} / \mathrm{cm}^{2}\right)$ & 0 \\
MR method & $\mathrm{HAMR}$ \\
Light - spot diameter $d_{\mathrm{L}}(\mathrm{nm})$ & 9.0 \\
Heat - spot diameter $d_{\mathrm{w}}(\mathrm{nm})$ & 10 \\
Linear velocity $v(\mathrm{~m} / \mathrm{s})$ & 10 \\
Thermal conductivity of IL1 $K(\mathrm{~W} /(\mathrm{cmK}))$ & 0.5 \\
Standard deviation of Curie temp. $\sigma_{T \mathrm{c}} / T_{\mathrm{c}}(\%)$ & 0 \\
\hline
\end{tabular}

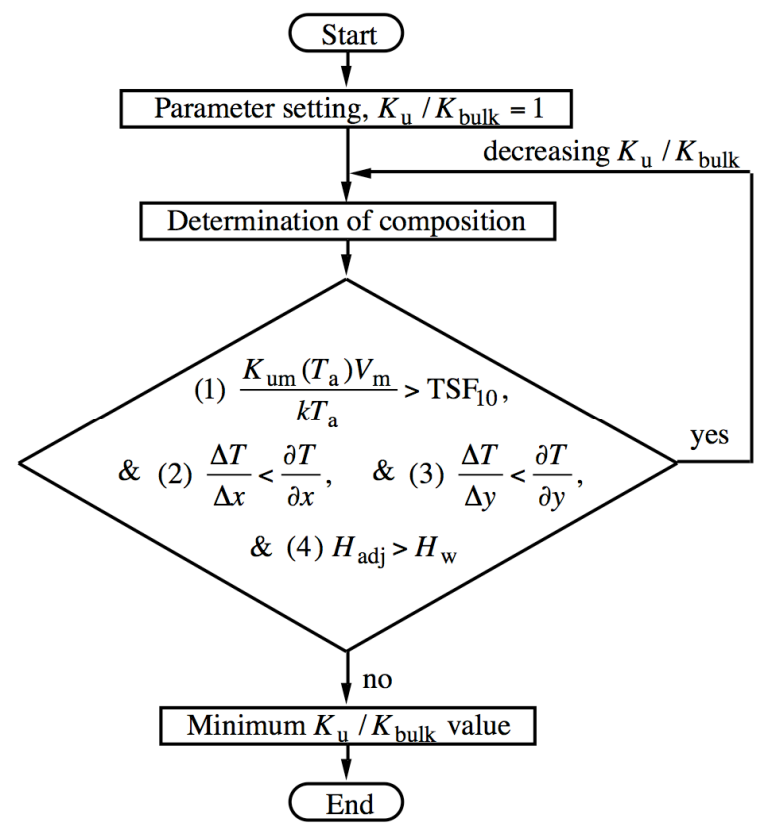

Fig. 4 HAMR media design procedure for obtaining the minimum anisotropy constant ratio $K_{\mathrm{u}} / K_{\text {bulk }}{ }^{3)}$.

Condition (1), the information stability during 10 years of archiving, is expressed as

$$
\frac{K_{\mathrm{um}}\left(T_{\mathrm{a}}\right) V_{\mathrm{m}}}{k T_{\mathrm{a}}} \geq \mathrm{TSF}_{10},
$$

where $K_{\mathrm{um}}\left(T_{\mathrm{a}}\right) V_{\mathrm{m}} / k T_{\mathrm{a}}$ is a medium thermal stability factor, $K_{\mathrm{um}}\left(T_{\mathrm{a}}\right)$ is the mean anisotropy constant,
$V_{\mathrm{m}}=D_{\mathrm{m}}{ }^{2} \times h$ is the grain volume for the mean grain size $D_{\mathrm{m}}, k$ is the Boltzmann constant, and $\mathrm{TSF}_{10}$ is the statistical thermal stability factor during 10 years of archiving ${ }^{3)}$ calculated statistically using grain-error probability and many bits under the condition of a $10^{-3}$ bit error rate. The statistical thermal stability factor $\operatorname{TSF}\left(\tau, n, \sigma_{\mathrm{D}}, \sigma_{\mathrm{K}}\right)$ is generally a function of the archiving period $\tau$, the grain number per bit $n$, the standard deviation of the grain size $\sigma_{\mathrm{D}}$, and the standard deviation of the anisotropy $\sigma_{\mathrm{K}}{ }^{5}$. TSF is unrelated to $K_{\mathrm{u}}$. And $\mathrm{TSF}_{10}$ means $\operatorname{TSF}\left(10\right.$ years, $\left.n, \sigma_{\mathrm{D}}, \sigma_{\mathrm{K}}\right)$.

Condition (2), the information stability on the trailing side during writing, is expressed as

$$
\frac{\Delta T}{\Delta x}=\frac{T_{\mathrm{w}}-T_{\mathrm{rec}}}{\Delta x} \leq \frac{\partial T}{\partial x},
$$

where $\Delta T / \Delta x$ is the medium thermal gradient for the down-track direction.

Condition (3), the information stability in adjacent tracks during rewriting, is expressed as

$$
\frac{\Delta T}{\Delta y}=\frac{T_{\mathrm{w}}-T_{\mathrm{adj}}}{\Delta y} \leq \frac{\partial T}{\partial y},
$$

where $\Delta T / \Delta y$ is the medium thermal gradient for the cross-track direction. $\Delta T / \Delta x$ and $\Delta T / \Delta y$ are the minimum thermal gradients required by the medium for information stability ${ }^{3}$.

Condition (4), the information stability under the main pole during rewriting, is expressed $a^{3)}$

$$
H_{\text {adj }} \geq H_{\mathrm{w}} .
$$

Conditions (2) and (3) can be combined as

$$
\frac{\Delta T}{\Delta x}=\frac{\Delta T}{\Delta y} \leq \frac{\partial T}{\partial x}=\frac{\partial T}{\partial y},
$$

since $\partial T / \partial x \approx \partial T / \partial y$. Condition (4) has margins for all the cases we examined. Therefore, the major limiting factors in the media design are condition (1) given by Eq. (1) ((1) $\left.K_{\mathrm{um}}\left(T_{\mathrm{a}}\right) V_{\mathrm{m}} / k T_{\mathrm{a}} \geq \mathrm{TSF}_{10}\right)$ and conditions (2) and (3) given by Eq. (5) (hereafter, shown as $\Delta T / \Delta x=\Delta T / \Delta y \equiv \Delta T / \Delta x(y) \quad, \quad \partial T / \partial x=\partial T / \partial y$ $\equiv \partial T / \partial x(y)$, and (2), (3) $\Delta T / \Delta x(y) \leq \partial T / \partial x(y))$.

\section{Calculation Results}

\subsection{Anisotropy constant ratio}

The dependence of the required $K_{\mathrm{u}} / K_{\text {bulk }}$ value on $T_{\mathrm{w}}$ is shown in Table 2. We extended the calculation range up to $T_{\mathrm{w}}=700 \mathrm{~K}$. The Curie temperature $T_{\mathrm{c}}$ is 4-8 K higher than $T_{\mathrm{w}}$. $T_{\mathrm{w}}$ is determined by the light power used for heating, and the $T_{\mathrm{c}}$ of the medium is determined by $T_{\mathrm{w}}$. If the light power alone is increased for a medium with the same $T_{\mathrm{c}}$, the written bits will be spread in the cross-track direction, and it becomes impossible to keep the track pitch constant. Therefore, 
$T_{\mathrm{c}}$ must be increased to increase $T_{\mathrm{w}}$. The magnetization $M_{\mathrm{s}}, K_{\mathrm{um}}$, the mean coercivity $H_{\mathrm{cm}}$, and $K_{\mathrm{um}} V_{\mathrm{m}} / k T$ at $300 \mathrm{~K}$ are also shown in the table. $H_{\mathrm{cm}}$ was assumed to be equal to the mean anisotropy field $H_{\mathrm{km}}=2 K_{\mathrm{um}} / M_{\mathrm{s}} \cdot \mathrm{TSF}_{10}$ in condition (1) is constant for $T_{\mathrm{w}}$, and $K_{\mathrm{um}}\left(T_{\mathrm{a}}\right) V_{\mathrm{m}} / k T_{\mathrm{a}}$ increases as $T_{\mathrm{w}}$ increases since $K_{\mathrm{um}}\left(T_{\mathrm{a}}\right)$ increases ${ }^{4)} . \partial T / \partial x(y)$ in conditions (2) and (3) also increases as $T_{\mathrm{w}}$ increases $^{3)}$. The limiting factors are conditions $(2)$ and (3). $H_{\text {adj }}$ in condition (4) is sufficiently larger than $H_{\mathrm{w}}$. Although $K_{\text {um }}(300 \mathrm{~K})$ apparently increases as $T_{\text {w }}$ increases, $K_{\mathrm{u}} / K_{\text {bulk }}$ can decrease from 0.66 for $T_{\mathrm{w}}=500 \mathrm{~K}$ to 0.45 for $T_{\mathrm{w}}=700 \mathrm{~K}$. The optimum $d_{\mathrm{B}}, d_{\mathrm{T}}$, and $d_{\mathrm{T}} / d_{\mathrm{B}}$ values are shown in the table.

$K_{\mathrm{u}} / K_{\text {bulk }}$ is a function of the heat-transfer thermal gradient $\partial T / \partial x(y)$ as shown in Fig. 5. As $\partial T / \partial x(y)$ increases, $K_{\mathrm{u}} / K_{\text {bulk }}$ first becomes lower, and then becomes constant with respect to $\partial T / \partial x(y)$. In the range where $K_{\mathrm{u}} / K_{\text {bulk }}$ changes, the limiting factors are conditions (2) and (3) $\Delta T / \Delta x(y) \leq \partial T / \partial x(y)$. And it is condition (1) $K_{\mathrm{um}}\left(T_{\mathrm{a}}\right) V_{\mathrm{m}} / k T_{\mathrm{a}} \geq \mathrm{TSF}_{10}$ in the range where $K_{\mathrm{u}} / K_{\text {bulk }}$ is constant. Although increasing $T_{\mathrm{w}}$ is effective for reducing $K_{\mathrm{u}} / K_{\text {bulk }}$, a higher $\partial T / \partial x(y)$ is needed to obtain a lower $K_{\mathrm{u}} / K_{\text {bulk }}$. The filled circles show the $K_{\mathrm{u}} / K_{\text {bulk }}$ values and their $\partial T / \partial x(y)$ values calculated by a heat-transfer simulation. The values correspond to those in Table 2. If we can realize a greater increase in $\partial T / \partial x(y)$ by examining the media structure, we can expect to reduce $K_{\mathrm{u}} / K_{\text {bulk }}$ even further.

Table 2 Calculation results of HAMR media design for various writing temperatures $T_{\mathrm{w}}$.

\begin{tabular}{l|ccc}
\hline$T_{\mathrm{w}}(\mathrm{K})$ & 500 & 600 & 700 \\
\hline$D_{\mathrm{m}}(\mathrm{nm})$ & 4.92 & 4.92 & 4.92 \\
$T_{\mathrm{c}}(\mathrm{K})$ & 508 & 606 & 704 \\
$M_{\mathrm{s}}(300 \mathrm{~K})\left(\mathrm{emu} / \mathrm{cm}^{3}\right)$ & 616 & 771 & 912 \\
$K_{\mathrm{um}}(300 \mathrm{~K})\left(10^{6} \mathrm{erg} / \mathrm{cm}^{3}\right)$ & 17 & 21 & 26 \\
$H_{\mathrm{cm}}(300 \mathrm{~K})=H_{\mathrm{km}}(300 \mathrm{~K})(\mathrm{kOe})$ & 54 & 55 & 57 \\
$K_{\mathrm{um}} V_{\mathrm{m}} / k T(300 \mathrm{~K})$ & 78 & 99 & 120 \\
\hline $\mathrm{TSF}_{10}$ & 62 & 62 & 62 \\
$(1) K_{\mathrm{um}}\left(T_{\mathrm{a}}\right) V_{\mathrm{m}} / k T_{\mathrm{a}} \geq \mathrm{TSF}_{10}$ & 64 & 84 & 105 \\
\hline$\partial T / \partial x(y)(\mathrm{K} / \mathrm{nm})$ & 6.9 & 11.0 & 15.1 \\
$(2),(3) \Delta T / \Delta x(y)(\mathrm{K} / \mathrm{nm})$ & 6.9 & 11.0 & 15.1 \\
\multicolumn{1}{c|}{$\leq \partial T / \partial x(y)$} & & & \\
\hline$H_{\mathrm{w}}(\mathrm{kOe})$ & 9.85 & 12.3 & 14.6 \\
$(4) H_{\mathrm{adj}}(\mathrm{kOe}) \geq H_{\mathrm{w}}$ & 16.7 & 22.0 & 26.3 \\
\hline$K_{\mathrm{u}} / K_{\mathrm{bulk}}$ & 0.66 & 0.52 & 0.45 \\
\hline$d_{\mathrm{B}}(\mathrm{nm})$ & 6.57 & 6.81 & 6.95 \\
$d_{\mathrm{T}}(\mathrm{nm})$ & 21.3 & 20.6 & 20.1 \\
$d_{\mathrm{T}} / d_{\mathrm{B}}$ & 3.25 & 3.02 & 2.90 \\
\hline
\end{tabular}

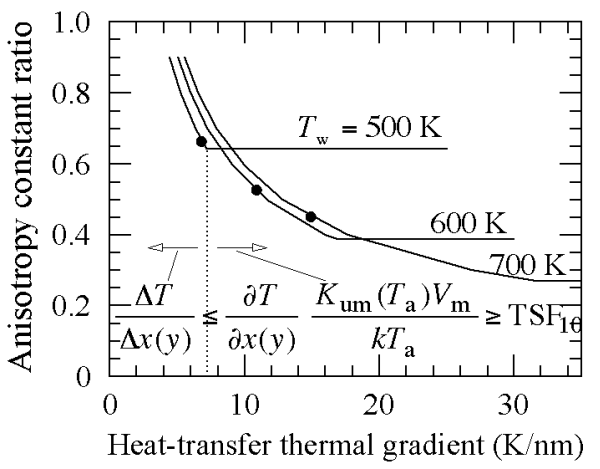

Fig. 5 Dependence of anisotropy constant ratio $K_{\mathrm{u}} / K_{\text {bulk }}$ on heat-transfer thermal gradient $\partial T / \partial x(y)$ for various writing temperatures $T_{\mathrm{w}}$.

\subsection{RL thickness}

The dependence of $K_{\mathrm{u}} / K_{\text {bulk }}$ on $\partial T / \partial x(y)$ is shown in Fig. 6 where the calculation parameter is the RL thickness $h$. When (a) $T_{\mathrm{w}}=500 \mathrm{~K}, K_{\mathrm{u}} / K_{\text {bulk }}$, shown by the filled circles, is increased by changing $h$ from $8 \mathrm{~nm}$ to $6 \mathrm{~nm}$ as previously reported ${ }^{4)}$. On the other hand, when (b) $T_{\mathrm{w}}=700 \mathrm{~K}$, the $K_{\mathrm{u}} / K_{\text {bulk }}$ values are almost the same for the $h=6$ to $10 \mathrm{~nm}$ calculation range since the limiting factors become conditions (2) and (3) $\Delta T / \Delta x(y) \leq \partial T / \partial x(y)$ for $h=6$ $\mathrm{nm}$, and $\partial T / \partial x(y)$ is reduced by increasing $h$ due to the adiabatic effect of RL. Therefore, when $T_{\mathrm{w}}$ is high, a relatively thin $\mathrm{RL}$ thickness is allowable.

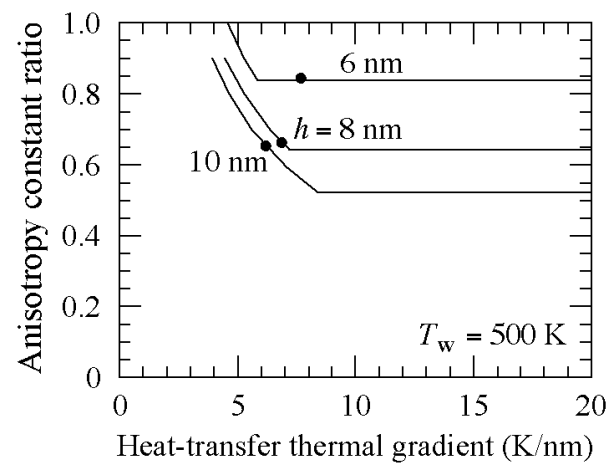

(a)

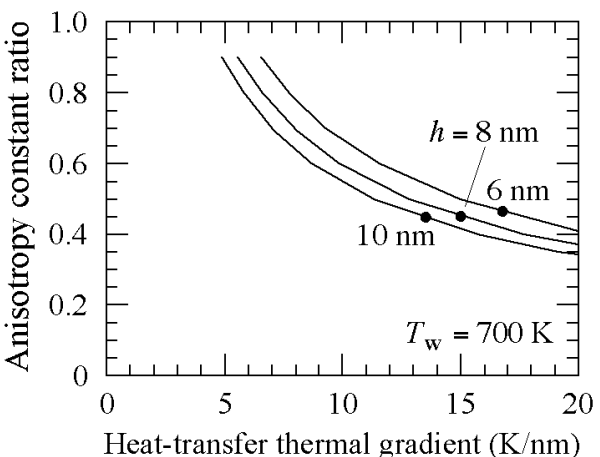

(b)

Fig. 6 Dependence of $K_{\mathrm{u}} / K_{\text {bulk }}$ on $\partial T / \partial x(y)$ at (a) $T_{\mathrm{w}}=500 \mathrm{~K}^{4)}$ and (b) $700 \mathrm{~K}$ for various $\mathrm{RL}$ thicknesses $h$. 


\subsection{Standard deviation of grain size}

The calculation results for the standard deviation of the grain size $\sigma_{\mathrm{D}}$ are shown in Fig. 7 .

As $\sigma_{\mathrm{D}}$ increases, $K_{\mathrm{u}} / K_{\text {bulk }}$, shown by the filled circle, is greatly increased when (a) $T_{\mathrm{w}}=500 \mathrm{~K}^{4)}$. On the other hand, when (b) $T_{\mathrm{w}}=700 \mathrm{~K}$, the increase in $K_{\mathrm{u}} / K_{\text {bulk }}$ caused by increasing $\sigma_{\mathrm{D}}$ is not great.

The limiting factor is largely (1) $K_{\mathrm{um}}\left(T_{\mathrm{a}}\right) V_{\mathrm{m}} / k T_{\mathrm{a}}$ $\geq \mathrm{TSF}_{10}$ when (a) $T_{\mathrm{w}}=500 \mathrm{~K}$. Figure 8 shows the dependence of the statistical thermal stability factor $\mathrm{TSF}_{10}$ on $\sigma_{\mathrm{D}}$ during $\tau=10$ years of archiving. As $\sigma_{\mathrm{D}}$ increases, the number of small size grains increases, and the probability of magnetization reversal for these small size grains is high over long periods, e.g. $\tau=10$ years. Therefore, $K_{\mathrm{um}}$ and $K_{\mathrm{u}} / K_{\text {bulk }}$ must be increased to maintain a low bit error rate when $\sigma_{\mathrm{D}}$ is large.

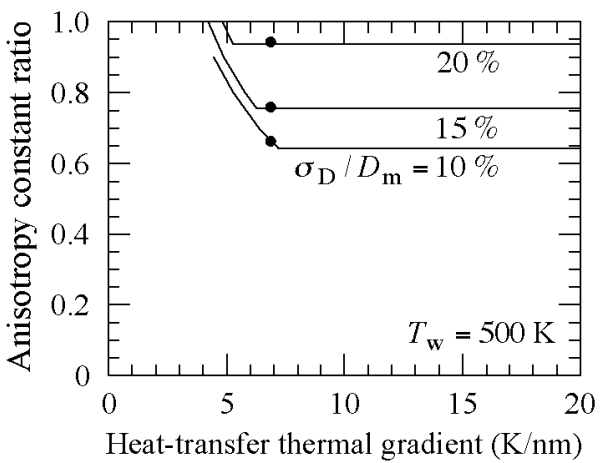

(a)

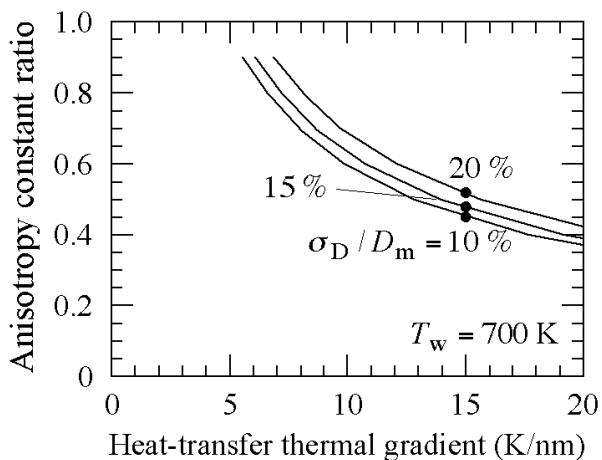

(b)

Fig. 7 Dependence of $K_{\mathrm{u}} / K_{\text {bulk }}$ on $\partial T / \partial x(y)$ at (a) $T_{\mathrm{w}}=500 \mathrm{~K}^{4)}$ and (b) $700 \mathrm{~K}$ for various standard deviations of grain size $\sigma_{\mathrm{D}} / D_{\mathrm{m}}$.

On the other hand, the limiting factor is (2), (3) $\Delta T / \Delta x(y) \leq \partial T / \partial x(y)$ when (b) $T_{\mathrm{w}}=700 \mathrm{~K} . \Delta T$ of $\Delta T / \Delta x$ is determined by the statistical thermal stability factor $\operatorname{TSF}_{\text {rec }}=\operatorname{TSF}\left(0.65 \mathrm{~ns}, n, \sigma_{\mathrm{D}}, \sigma_{\mathrm{K}}\right)$ for the writing period of $0.65 \mathrm{~ns}^{3}$. Figure 8 also shows the dependence of $\mathrm{TSF}_{\text {rec }}$ on $\sigma_{\mathrm{D}}$. The $\mathrm{TSF}_{\text {rec }}$ increase rate is smaller than that of $\mathrm{TSF}_{10}$ since the probability of magnetization reversal is low even for a small size grain during a short period, e.g. $0.65 \mathrm{~ns}$. Therefore, a large $\sigma_{\mathrm{D}}$ is allowable during the writing process. After writing, the medium is cooled to $T_{\mathrm{a}}$, and then $K_{\mathrm{um}}$ becomes sufficiently large to hold information for 10 years even when the grain size is small.

Figure 9 shows the temperature dependence of $K_{\text {um }}$ for $T_{\mathrm{w}}=500 \mathrm{~K}$ and $700 \mathrm{~K} . K_{\mathrm{u}} / K_{\text {bulk }}$ is 0.45 for both cases. $K_{\mathrm{u}}\left(T_{\mathrm{a}}\right)$ for $T_{\mathrm{w}}=700 \mathrm{~K}$ is sufficiently larger than that for $K_{\mathrm{um}}\left(T_{\mathrm{a}}\right) V_{\mathrm{m}} / k T_{\mathrm{a}}=\mathrm{TSF}_{10}$ since the temperature difference between $T_{\mathrm{a}}$ and $T_{\mathrm{w}}$ is sufficiently large. On the other hand, $K_{\mathrm{u}}\left(T_{\mathrm{a}}\right)$ for $T_{\mathrm{w}}=$ $500 \mathrm{~K}$ is insufficient for $K_{\mathrm{um}}\left(T_{\mathrm{a}}\right) V_{\mathrm{m}} / k T_{\mathrm{a}}=\mathrm{TSF}_{10}$.

With conventional magnetic recording, microwaveassisted magnetic recording, and bit-patterned media, the limiting factor is $K_{\mathrm{um}}\left(T_{\mathrm{a}}\right) V_{\mathrm{m}} / k T_{\mathrm{a}} \geq \mathrm{TSF}_{10}$, and $\sigma_{\mathrm{D}}$ must be restricted to a small value. With HAMR, a relatively large $\sigma_{\mathrm{D}}$ is allowable when $T_{\mathrm{w}}$ is high. This is the advantage of HAMR, which utilizes the change of temperature.

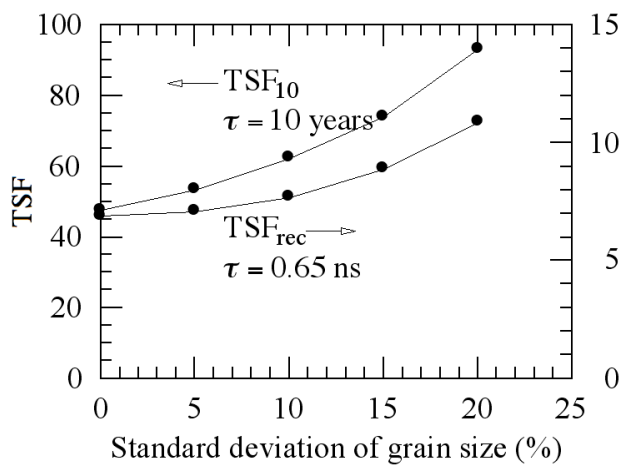

Fig. 8 Dependence of statistical thermal stability factor TSF on standard deviation of grain size $\sigma_{\mathrm{D}} / D_{\mathrm{m}}$ for a period $\tau=10$ years and $0.65 \mathrm{~ns}$.

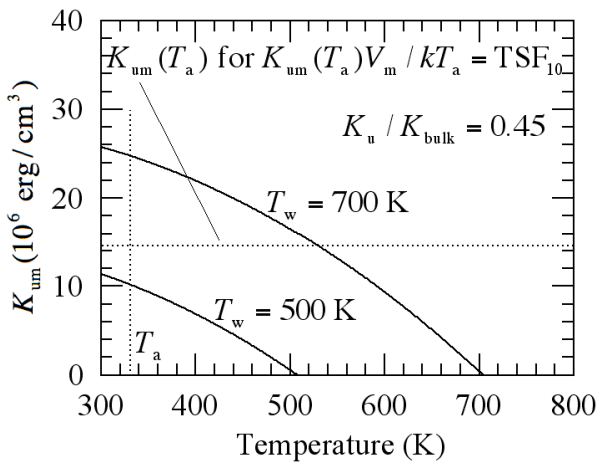

Fig. 9 Temperature dependence of $K_{\mathrm{um}}$ for $T_{\mathrm{w}}=500$ $\mathrm{K}$ and $700 \mathrm{~K}$.

\subsection{Standard deviation of anisotropy}

A normal distribution was used for the $K_{\mathrm{u}}$ distribution. The calculation results for the standard deviation of the anisotropy $\sigma_{\mathrm{K}}$ are shown in Fig. 10. A comparison of $\sigma_{\mathrm{D}}$ in Fig. 7 (a) and $\sigma_{\mathrm{K}}$ in Fig. 10 (a) reveals that the dependence of $K_{\mathrm{u}} / K_{\text {bulk }}$ on $\sigma_{\mathrm{K}}$ is smaller than that on $\sigma_{\mathrm{D}}$. This difference can be explained by the formula of the grain-error probability,

$$
1-\exp \left(-f_{0} \tau \exp \left(-\mathrm{TSF} \cdot \frac{D^{2}}{D_{\mathrm{m}}{ }^{2}} \cdot \frac{K_{\mathrm{u}}}{K_{\mathrm{um}}}\right)\right),
$$


where $f_{0}=10^{11} \mathrm{~s}^{-1}$ is an attempt frequency. In the parenthesis in the formula, the variables of grain size and anisotropy are $D^{2}$ and $K_{\mathrm{u}}$, respectively. Therefore, the dependence of $K_{\mathrm{u}} / K_{\text {bulk }}$ on $\sigma_{\mathrm{K}}$ is small.

As $\sigma_{\mathrm{K}}$ increases, $K_{\mathrm{u}} / K_{\text {bulk }}$, shown by the filled circle in Fig. 10, is greatly increased when (a) $T_{\mathrm{w}}=500$ $\mathrm{K}$. On the other hand, when (b) $T_{\mathrm{w}}=700 \mathrm{~K}$, there is little increase in $K_{\mathrm{u}} / K_{\text {bulk }}$ when $\sigma_{\mathrm{K}}$ is increased. The reason is the same as with the grain size distribution. Therefore, a relatively large $\sigma_{\mathrm{K}}$ is allowable when $T_{\mathrm{w}}$ is high, and this is also an advantage of HAMR.

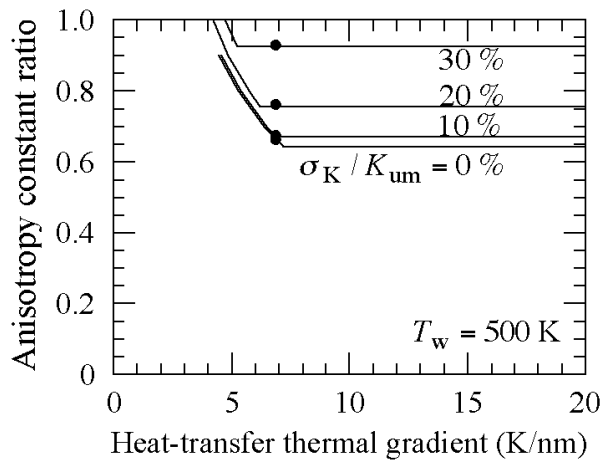

(a)

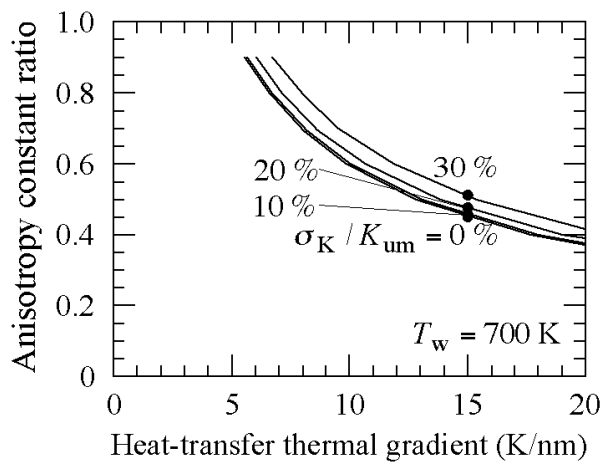

(b)

Fig. 10 Dependence of $K_{\mathrm{u}} / K_{\text {bulk }}$ on $\partial T / \partial x(y)$ at (a) $T_{\mathrm{w}}=500 \mathrm{~K}$ and (b) $700 \mathrm{~K}$ for various standard deviations of anisotropy $\sigma_{\mathrm{K}} / K_{\mathrm{um}}$.

\subsection{Standard deviation of Curie temperature}

Next, we discuss the $T_{\mathrm{c}}$ distribution. A normal distribution was used for the standard deviations of the writing temperature $\sigma_{T \mathrm{w}}$ and the Curie temperature $\sigma_{T \mathrm{c}}$. We assumed $n=1$. Figure 11 shows how $\sigma_{T \mathrm{w}}$ is calculated. The temperature at the center of the bit pitch $d_{\mathrm{B}}$ is $T_{\mathrm{w}}$, and the temperature at the edge is $T_{\mathrm{w}} \pm \Delta T_{\mathrm{w}}$. Then $\Delta T_{\mathrm{w}}$ is expressed as

$$
\Delta T_{\mathrm{w}}=\frac{\partial T}{\partial x} \cdot \frac{d_{\mathrm{B}}}{2} .
$$

If $\Delta T_{\mathrm{w}}=3.29 \sigma_{T \mathrm{w}}$, the bit error rate of the write error becomes $10^{-3}$, that is,

$$
\int_{-3.29 \sigma}^{3.29 \sigma} f(x) d x=1-10^{-3}
$$

where $f(x)$ is a normal distribution. Therefore, $\sigma_{T w}$ is expressed as

$$
\sigma_{T \mathrm{w}}=\frac{1}{3.29} \cdot \frac{\partial T}{\partial x} \cdot \frac{d_{\mathrm{B}}}{2} .
$$

$\sigma_{T \mathrm{w}} \approx \sigma_{T \mathrm{c}}$ is assumed, and the calculation results are summarized in Table 3. As $T_{\mathrm{w}}$ increases, $\partial T / \partial x$ increases, and then, $\sigma_{T \mathrm{c}}$ increases. $\sigma_{T \mathrm{c}} / T_{\mathrm{c}}$ also increases despite the increase in $T_{\mathrm{c}}$. Therefore, when $T_{\mathrm{w}}$ is high, a relatively large $\sigma_{T_{\mathrm{c}}} / T_{\mathrm{c}}$ is allowable.

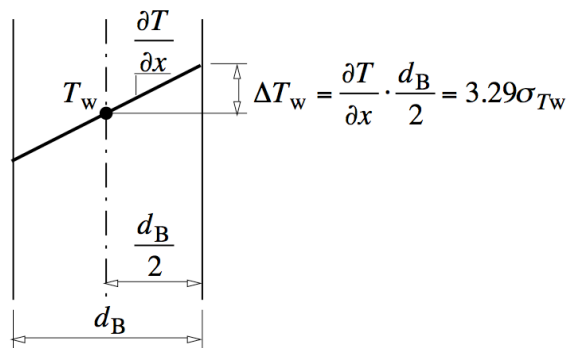

Fig. 11 Standard deviation of writing temperature $\sigma_{T \mathrm{w}}$ for a bit error rate of $10^{-3}$.

Table 3 Dependence of standard deviation of Curie temperature $\sigma_{T \mathrm{c}} / T_{\mathrm{c}}$ on writing temperature $T_{\mathrm{w}}$.

\begin{tabular}{l|ccc}
\hline$T_{\mathrm{w}}(\mathrm{K})$ & 500 & 600 & 700 \\
$n$ (grain $/$ bit) & 1 & 1 & 1 \\
\hline$\partial T / \partial x(\mathrm{~K} / \mathrm{nm})$ & 6.92 & 11.0 & 15.1 \\
$d_{\mathrm{B}}(\mathrm{nm})$ & 6.57 & 6.81 & 6.95 \\
$\sigma_{T \mathrm{w}}(\mathrm{K})$ & 6.91 & 11.4 & 15.9 \\
\hline$\sigma_{T \mathrm{c}} \approx \sigma_{T \mathrm{w}}(\mathrm{K})$ & 6.91 & 11.4 & 15.9 \\
$T_{\mathrm{c}}(\mathrm{K})$ & 508 & 606 & 704 \\
$\sigma_{T \mathrm{c}} / T_{\mathrm{c}}(\%)$ & 1.4 & 1.9 & 2.3 \\
\hline
\end{tabular}

3.6 Temperature dependence of anisotropy field

The temperature dependence of the magnetic properties is important in $\mathrm{HAMR}^{6}$. Figure 12 (a) shows the temperature dependence of the normalized anisotropy field $H_{\mathrm{k}} / H_{\mathrm{k}}(300 \mathrm{~K})$ for a low $T_{\mathrm{c}}=580 \mathrm{~K}$. The solid line was calculated using a mean field theory. The filled circles are experimental data for $\mathrm{Fe}-\mathrm{Ni}-\mathrm{Pt}$ reported by Thiele et al. ${ }^{7}$. The values of the experimental data are somewhat lower than those predicted by the theory. We think that this experimental behavior is a disadvantage of HAMR in terms of the writing field range for a good signal-to-noise ratio ${ }^{6}$. In contrast, the experimental data are well fitted to the calculation line for a high $T_{\mathrm{c}}$ $=770 \mathrm{~K}$ as shown in Fig. $12(\mathrm{~b})$. Since $T_{\mathrm{w}}$ must be increased to increase $T_{\mathrm{c}}$, increasing $T_{\mathrm{w}}$ also has an advantage in terms of the temperature dependence of the anisotropy field. 


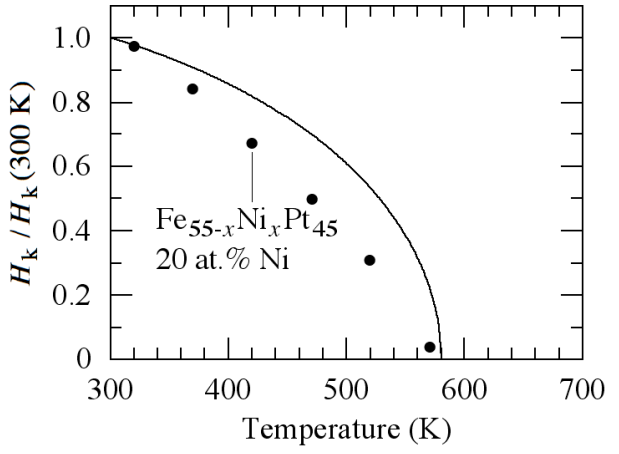

(a)

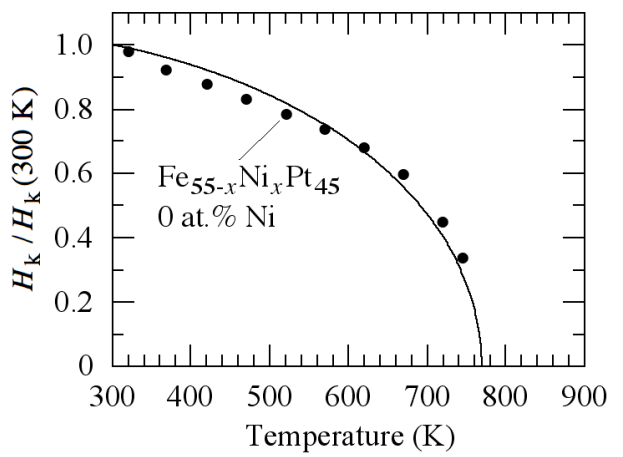

(b)

Fig. 12 Temperature dependence of normalized anisotropy field $H_{\mathrm{k}} / H_{\mathrm{k}}(300 \mathrm{~K})$ for (a) Curie temperature $T_{\mathrm{c}}=580 \mathrm{~K}$ and (b) $770 \mathrm{~K}$ (filled circles: $\mathrm{Fe}-\mathrm{Ni}-\mathrm{Pt} \operatorname{data}^{7)}$ ).

\section{Conclusions}

We summarized the advantages of increasing the writing temperature $T_{\mathrm{w}}$ in heat-assisted magnetic recording (HAMR).

(1) Since $K_{\mathrm{um}}\left(T_{\mathrm{a}}\right)$ and $\partial T / \partial x(y)$ are increased, the anisotropy constant ratio $K_{\mathrm{u}} / K_{\text {bulk }}$ can be decreased where $K_{\mathrm{um}}\left(T_{\mathrm{a}}\right)$ is the mean anisotropy constant at the maximum working temperature $T_{\text {a }}$ of the hard drive, and $\partial T / \partial x(y)$ is the heat-transfer thermal gradient calculated by a heat-transfer simulation.
(2) The $K_{\mathrm{u}} / K_{\text {bulk }}$ values are almost the same for the calculation range from the recording-layer $(\mathrm{RL})$ thickness $h=6 \mathrm{~nm}$ to $10 \mathrm{~nm}$ since $\partial T / \partial x(y)$ is reduced by increasing $h$ due to the adiabatic effect of RL. Therefore, a relatively thin RL thickness is allowable.

(3) A relatively large standard deviation of grain size $\sigma_{\mathrm{D}}$ is allowable since the probability of magnetization reversal is low even for small size grains during the writing period.

(4) A relatively large standard deviation of anisotropy $\sigma_{\mathrm{K}}$ is allowable.

(5) A relatively large standard deviation of Curie temperature $\sigma_{T c}$ is allowable since $\partial T / \partial x(y)$ is increased. $\sigma_{T \mathrm{c}} / T_{\mathrm{c}}$ also increases despite the increase in the Curie temperature $T_{\mathrm{c}}$.

(6) For Fe-Ni-Pt films, the temperature dependence of the anisotropy field is suitable for HAMR when $T_{\mathrm{w}}$ is high.

All the above-mentioned factors are advantageous when preparing HAMR media.

Acknowledgements We acknowledge the support of the Advanced Storage Research Consortium (ASRC), Japan.

\section{References}

1) S. H. Charap, P. -L. Lu, and Y. He: IEEE Trans. Magn., 33 , 978 (1997).

2) T. Kobayashi, Y. Isowaki, and Y. Fujiwara: J. Magn. Soc. Jpn., 39, 8 (2015).

3) T. Kobayashi, Y. Isowaki, and Y. Fujiwara: J. Magn. Soc. Jpn., 39, 139 (2015).

4) T. Kobayashi, Y. Isowaki, and Y. Fujiwara: to be published in J. Magn. Soc. Jpn., 40, (2016).

5) Y. Isowaki, T. Kobayashi, and Y. Fujiwara: J. Magn. Soc. Jpn., 38, 1 (2014).

6) J. -G. Zhu and H. Li: IEEE Trans. Magn., 49, 765 (2013)

7) J. -U. Thiele, K. R. Coffey, M. F. Toney, J. A. Hedstrom, and A. J. Kellock: J. Appl. Phys., 91, 6595 (2002).

Received Oct. 9, 2015; Accepted Dec. 25, 2015 